\title{
USG Guided Forearm Nerve Block
}

\author{
Madhuri Dadke ${ }^{1}$, Sandeep Diwan ${ }^{1}$ \\ ${ }^{1}$ Department of Anaesthesia, Sancheti Hospital, Pune, Maharashtra, India.
}

\section{Introduction}

Forearm blocks (FAB) are implemented to provide anaesthesia for hand surgeries, as a rescue block for failed or patchy proximal brachial plexus block and to provide postoperative analgesia after a regional or a general anaesthetic [1]. The advantage of these distal blocks is preservation of proximal motor function and avoidance of central structures such as the pleura, subclavian or axillary artery and the phrenic nerve. The proximal muscle function is preserved with these blocks, requiring minimal doses of local anaesthetic. These blocks can be implemented in outpatients [2] and with patients on anticoagulants [3]. Distal nerve blocks do not prevent tourniquet pain since the lateral antebrachial cutaneous nerve of forearm (musculocutaneous nerve), the medial cutaneous nerve of the arm, the posterior cutaneous nerve of the arm, and the intercostobrachial nerve that provide cutaneous innervation of the upper arm are not blocked [4]. The above mentioned nerves are blocked in the arm. Blockade of multiple nerves are needed and so involves multiple injections that may cause more patient discomfort.

\section{Equipment and Scan Technique}

The equipment recommended for a forearm block includes the following:

1. Ultrasound machine with a linear transducer (8-14Mhz), sterile sleeve, and sterile gel

2. Standard nerve block tray

3. Syringes

4. Local anesthetic drug

5. Insulated stimulating needle $5 \mathrm{~cm}$

6. Peripheral nerve stimulator

7. Sterile gloves

8. Pressure monitoring

\section{Scanning Technique}

The arm is in supine position (Figure 1)

\section{Anatomy}

\section{The Median Nerve course}

The median nerve is located at the level of the elbow crease by ultrasound in short axis with a linear transducer [5]. Initially the brachial artery is located, and the median nerve appears as a hyperechoic oval structure on the medial side of the brachial artery. Scanning distally to the elbow crease (Figure 2) till the mid forearm, the median nerve separates from the artery and lies deep to the pronator teres muscle and the flexor digitorum superficialis.

\section{The Median Nerve : Proximal forearm block technique}

With the arm abducted and supinated, the skin is disinfected and the transducer positioned transversely in the crease. The median nerve should be identified on the medial side of the artery. Sliding the transducer distally (Figure 3) we follow the median nerve till the proximal third of the forearm until the nerve is sandwiched between the flexor digitorum superficialis and profundus (Figure 4). The needle is inserted in-plane from lateral to medial side of the transducer (Figure 3). After negative aspiration, 4-5 mL oflocal anesthetic is injected (Figure 5).

Address of Correspondence: Dr. Sandeep Diwan, Consultant Anaesthesiologist, Sancheti Hospital, Pune, Maharashtra, India.

E-mail: sdiwan14@gmail.com

DOI: 10.13107/ijra.2021.v02i01.030 | C 2021 International Journal of Regional Anaesthesia | Available on www.ijrajournal.com | This is an Open Access article distributed under the terms of the Creative Commons Attribution Non-Commercial License (http://creativecommons.org/licenses/by-nc/3.0) which permits unrestricted non-commercial use, distribution, and reproduction in any medium, provided the original work is properly cited.

How to cite this article: Dadke M, Diwan S | USG Guided Forearm Nerve Block | International Journal of Regional Anaesthesia | JanuaryJune $2021 ; 2(1): 72-77$. 


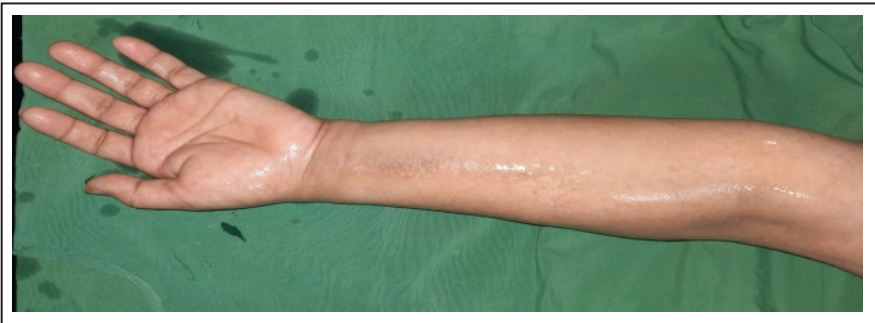

Figure 1: Left forearm in supine position

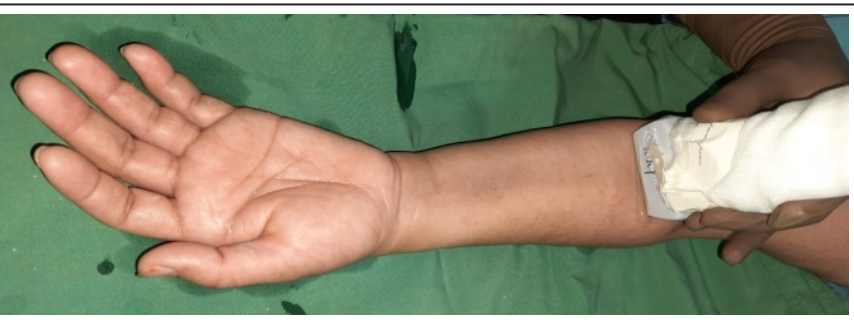

Figure 2: Axial placement of linear probe in proximal area of left forearm

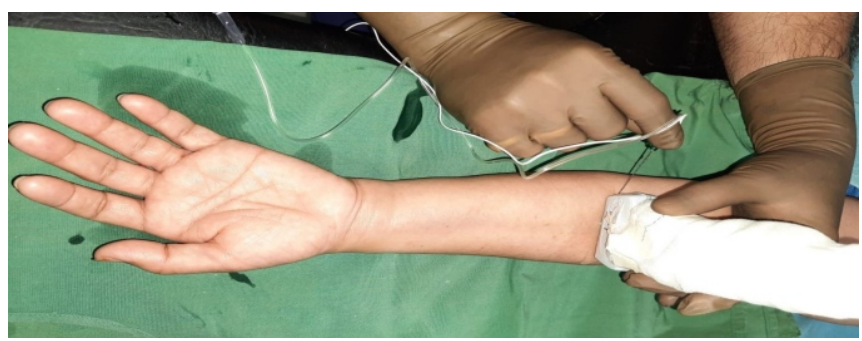

Figure 3: In plane needle placement beneath the linear probe for left median nerve block

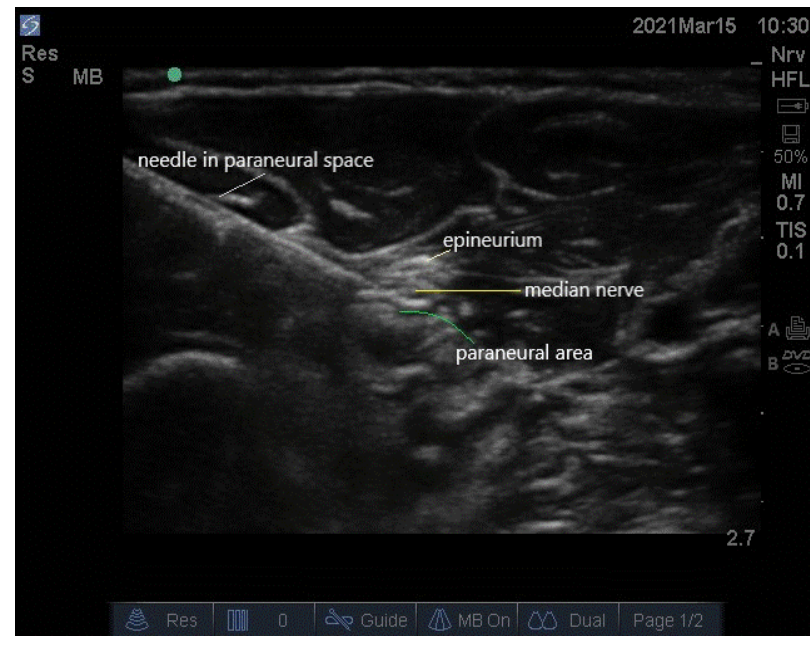

Figure 4: Median nerve in Axial scan with needle tip in close approximation of the median nerve

\section{The Ulnar Nerve course}

The ulnar nerve exits from the ulnar notch at the posteromedial aspect of the elbow and can be located by a linear transducer in short axis, as a round hyperechoic structure, after which it enters the forearm underneath the flexor carpi ulnaris [6].

The nerve is relatively immobile within the ulnar sulcus and is at risk of needle trauma if an injection is made here, and this can cause pressure induced neuropraxia so this approach must be avoided.

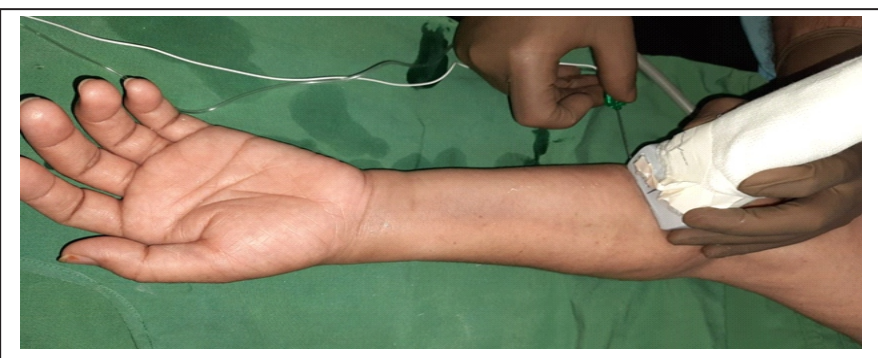

Figure 6: In-plane needle placement beneath linear probe for left ulnar nerve block

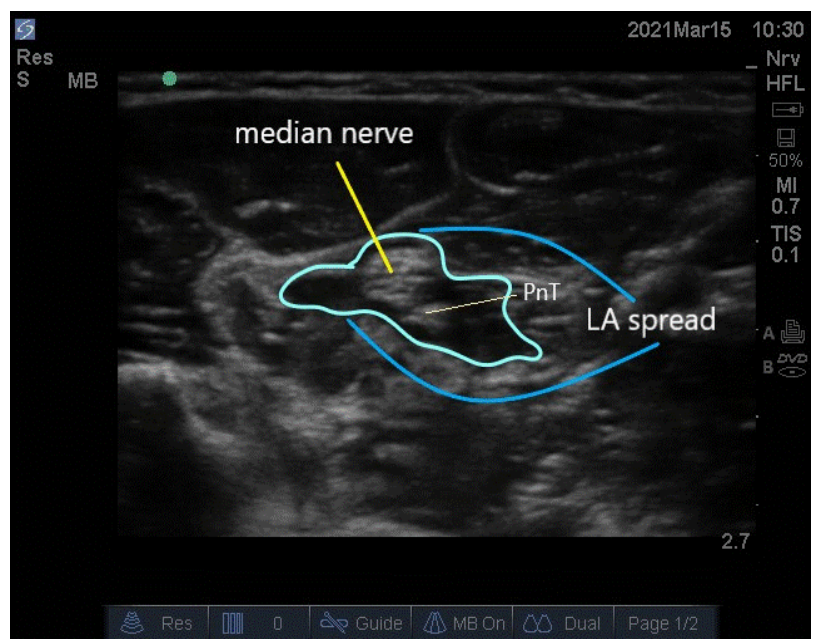

Figure 5: LA surrounds the media nerve; PnT - paraneural tissue
The Ulnar Nerve : Proximal forearm block technique

With the transducer positioned on the medial aspect of the forearm (Figure 6), the ulnar artery is located and medial to that the honeycomb appearance of the ulnar nerve is identified [7]. At this level needle is inserted in-plane from medial to lateral beneath the transducer (Figure 6, 7). After negative aspiration, $4-5 \mathrm{~mL}$ of local anesthetic is injected.

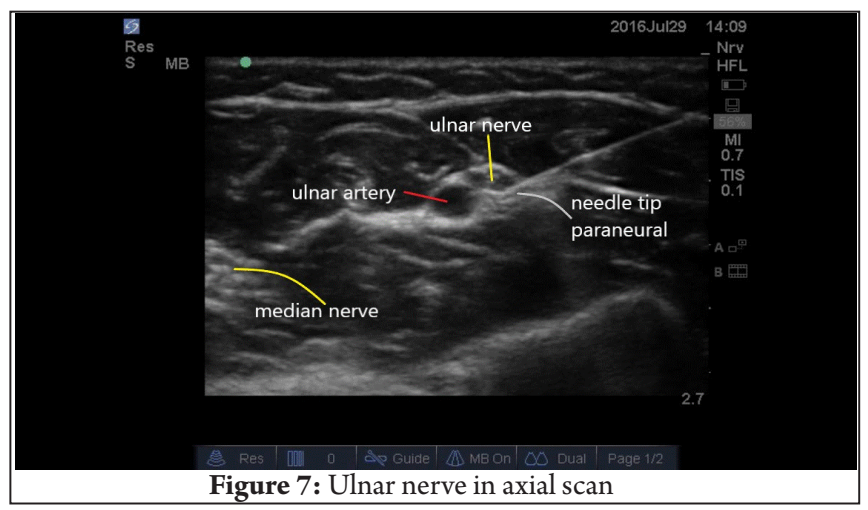

73 | International Journal of Regional Anaesthesia | Volume 2 | Issue 1 | January-June 2021 | Page $72-77$ 


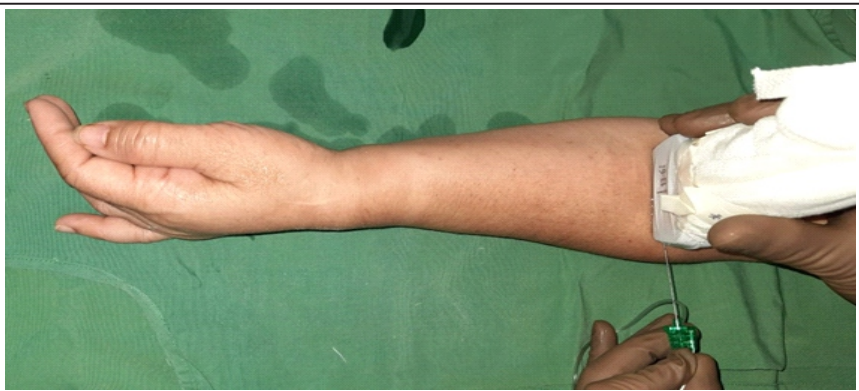

Figure 8: In-plane needle placement beneath linear probe for left superficial radial nerve block, with the forearm in semi-prone position

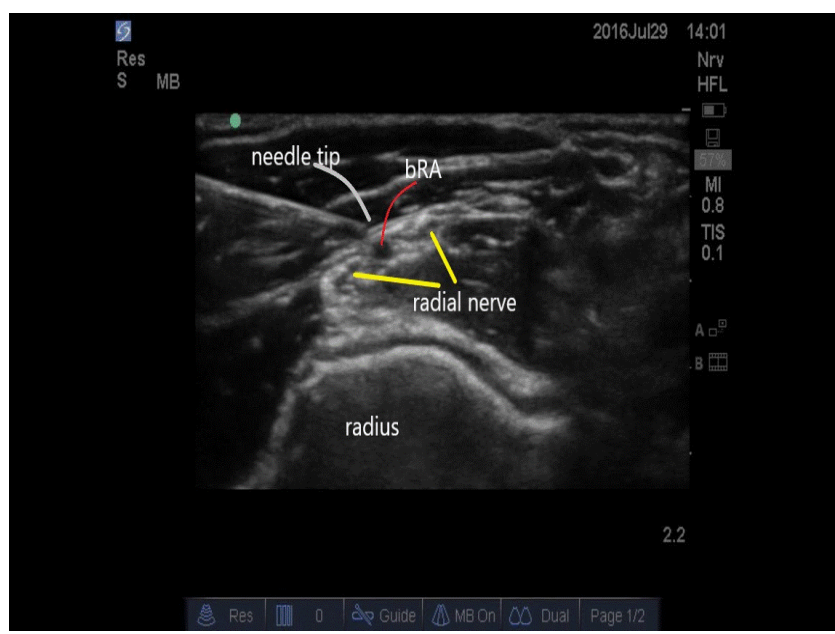

Figure 9: Radial nerve in axial scan

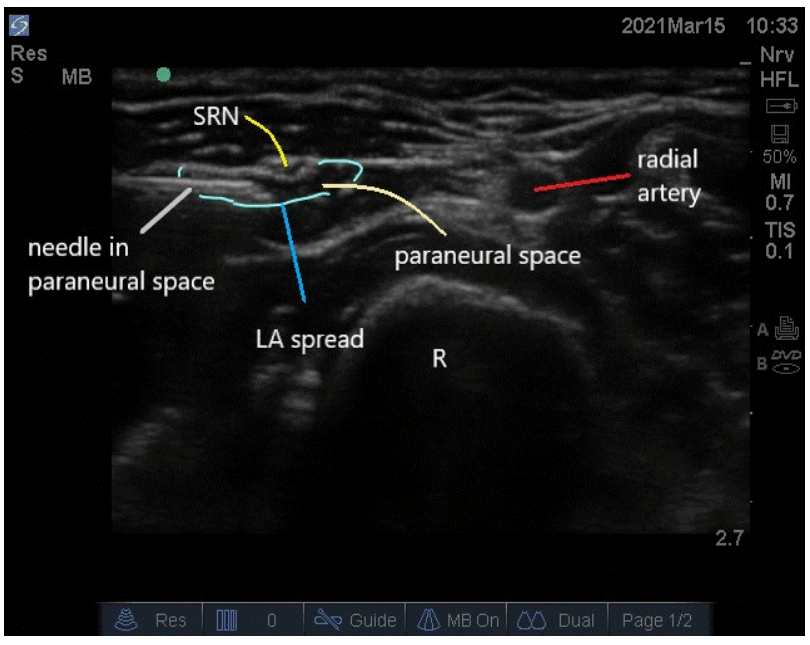

Figure 10: The superficial radial nerve (SRN) engulfed by LA (local anaesthetic)

\section{The Radial Nerve course}

To visualise the radial nerve the transducer is placed transversely on the anterolateral aspect of the distal arm, 3-4 cm above the elbow crease. At this point it lies between the brachioradialis and the brachialis muscles. The nerve appears as a hyperechoic, triangular or oval structure with the characteristic honeycomb appearance of a distal peripheral nerve [7]. The nerve divides just distal to the elbow crease into the superficial (sensory) and deep (motor) branches. These smaller divisions of the radial nerve are more challenging to identify in the forearm; therefore, a single injection at or just above the elbow crease is favored because it ensures blockade of both. For more discreet blocks of the superficial (Figure 10) and posterior interosseus nerves the transducer is slid caudal to visualise these nerves in the forearm.

\section{The Radial Nerve: Proximal forearm block}

The arm is kept abducted and pronated (Figure 8). Once the skin is disinfected, the transducer is positioned anterior to the elbow joint. The radial nerve is visualized between the extensor carpi radialis muscles and brachioradialis. The needle is inserted in plane and its tip placed next to the radial nerve (Figure 9). With nerve stimulator a wrist or finger extension response should be elicited when the needle is within proximity of the nerve. After negative aspiration, $4-5 \mathrm{~mL}$ of local anesthetic is injected [8] (Figure 10).

\section{Blockade of cutaneous nerves}

All forearm cutaneous nerves can be located in the mid and distal arm :

1) Lateral antebrachial cutaneous nerve ( $\mathrm{LABCN})$,

2) Medial antebrachial cutaneous nerve ( $M A B C N)$

3) Posterior antebrachial cutaneous nerve (PABCN)

\section{The lateral antebrachial cutaneous nerve ( $\mathrm{LABCN})$}

The musculocutaneous nerve pierces the deep fascia lateral to biceps brachii to emerge lateral to the biceps tendon and brachioradialis. It continues into the forearm as the lateral cutaneous nerve and provides sensory innervation to the lateral aspect of the forearm. This nerve initially enters the deep forearm, but then pierces the deep fascia to become subcutaneous. In this region, it can be found close to the cephalic vein [9]. Posterior antebrachial cutaneous nerve (PABCN); $\mathrm{MCN}$ musculocutaneous nerve; branch of Posterior antebrachial cutaneous nerve (b-PABCN).

\section{The LABCN Block}

The linear transducer is placed in mid axilla (Figure 13) to identify the MCN (Figure 11) and followed distally anterolaterally to trace the LABCN (Figure 12). The needle is inserted in plane lateral to medial and 3 ml of LA injected. 


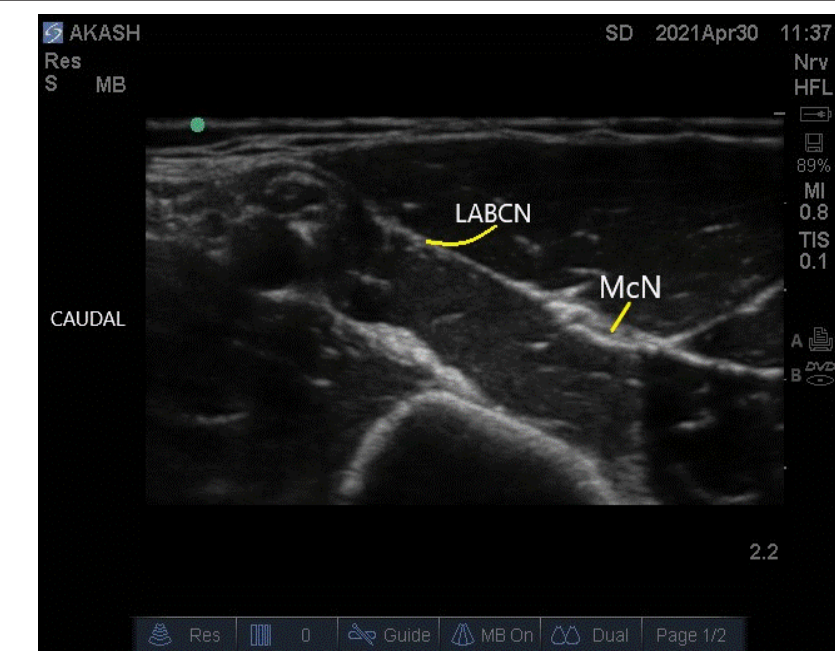

Figure 11: The musculocutaneous nerve $(\mathrm{MCN})$ in axial scan

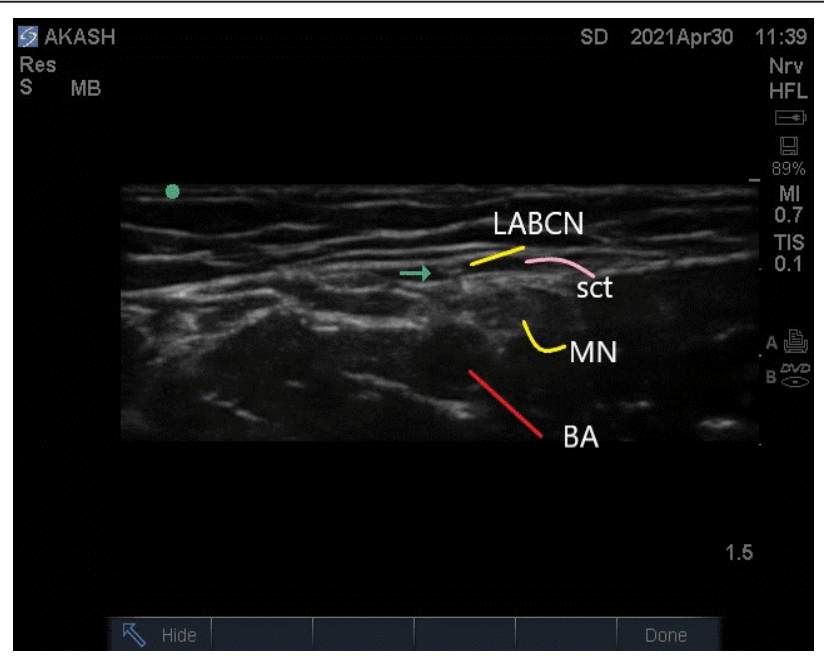

Figure 12: $\mathrm{LABCN}$ seen in the subcutaneous tissue (sct). $\mathrm{BA}=\mathrm{Brachial}$ artery, $\mathrm{MN}=$ Median Nerve.

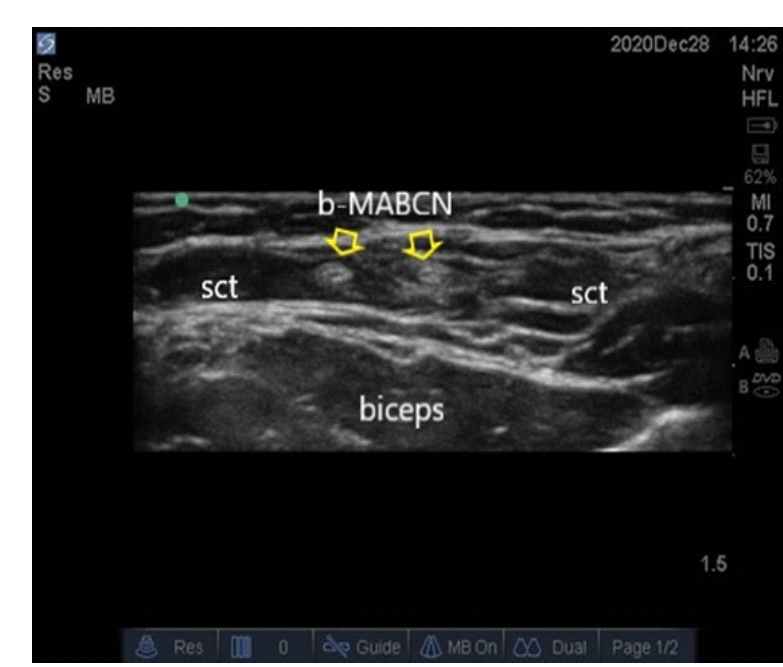

Figure 14: Medial antebrachial cutaneous nerve in the subcutaneous tissue; sct-subcutaneous tissue; branches of medial antebrachial cutaneous nerve (b-MABCN)

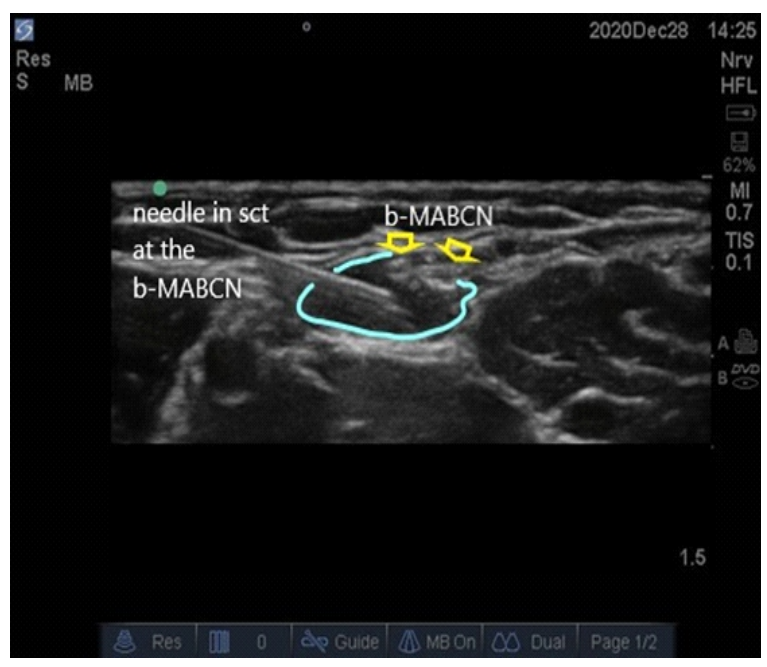

Figure 15: sct-subcutaneous tissue; branches of medial antebrachial cutaneous nerve (b-MABCN) 


\section{The PABCN Block}

Keeping the arm adducted and internally rotated with elbow flexed and arm on chest (Figure 16), the linear transducer is placed in short axis to identify the radial nerve. Tracing it distally and posteriorly the small hyperechoic $\mathrm{PABCN}$ is seen emerging to become superficial and dividing into branches. Needle is inserted in plane lateral to medial approach to block to inject the LA (Figure 17); (Figure 18-20).
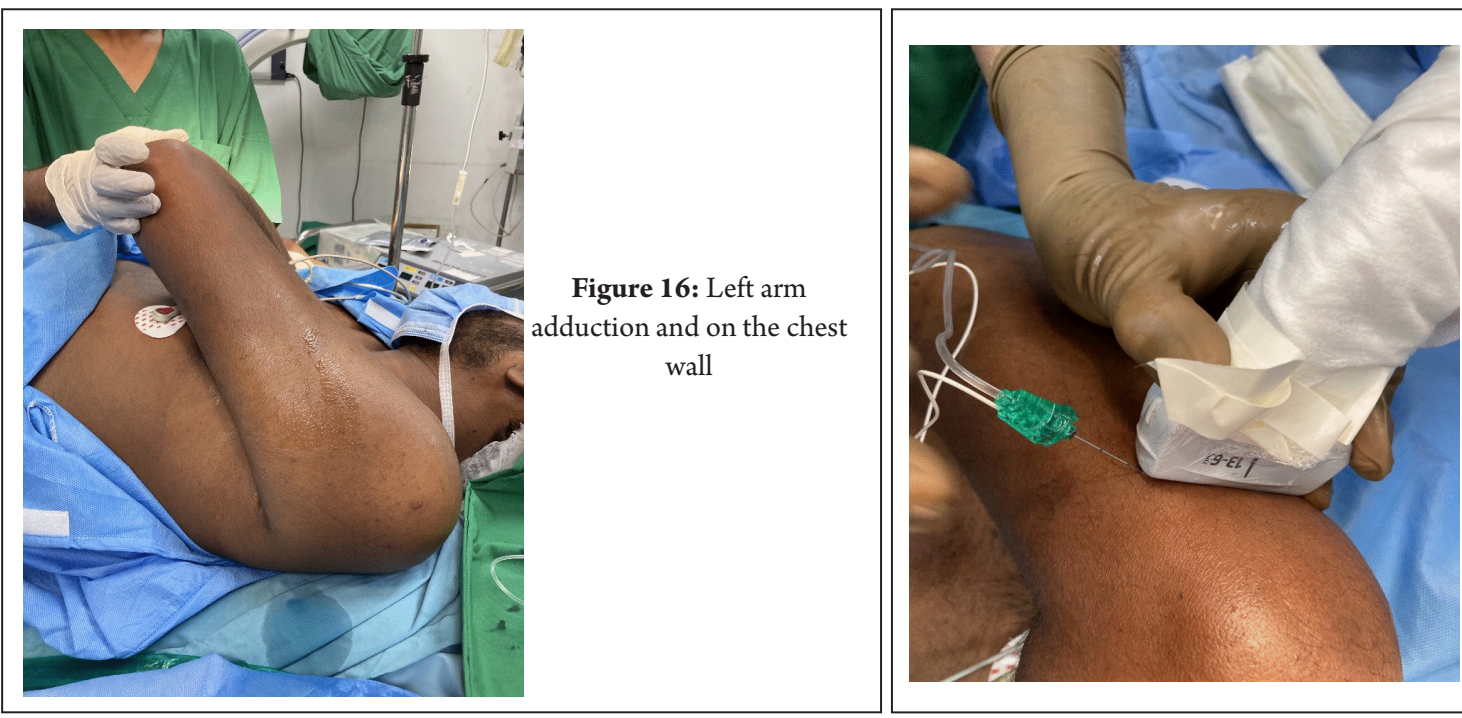

Figure 17: Needle from lateral to medial for $\mathrm{PABCN}$

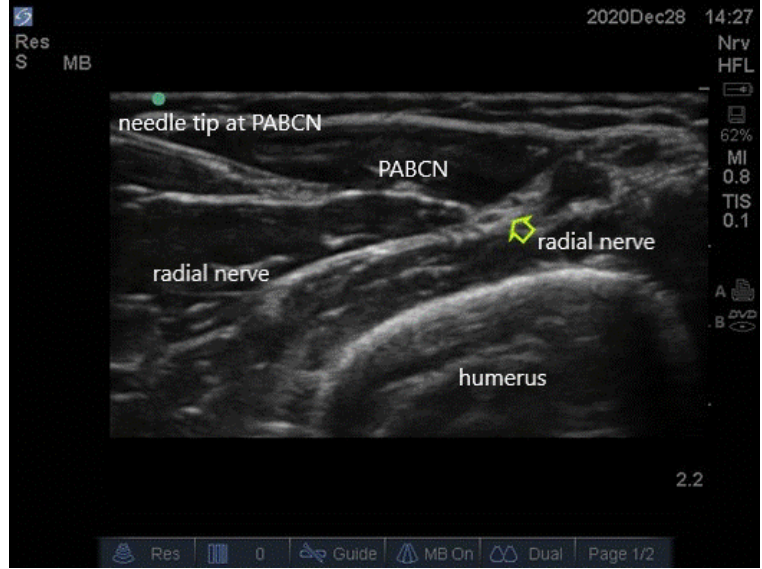

Figure 18: Needle tip at PABCN. Posterior antebrachial cutaneous nerve $(\mathrm{PABCN})$

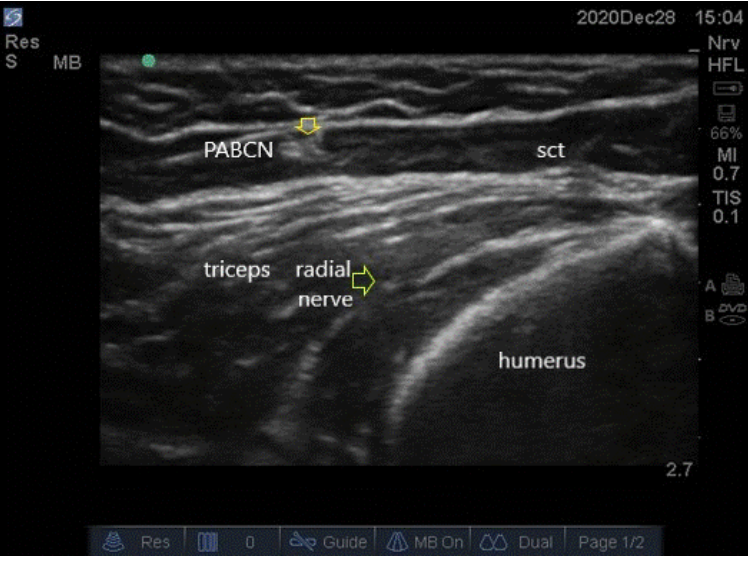

Figure 19: Distal arm : $\mathrm{PABCN}$ in the subcutaneous tissue; sctsubcutaneous tissue. Posterior antebrachial cutaneous nerve (PABCN)
Figure 20: The posterior antebrachial cutaneous nerve(PABCN) branching off the Radial Nerve( $\mathrm{RN}$ )

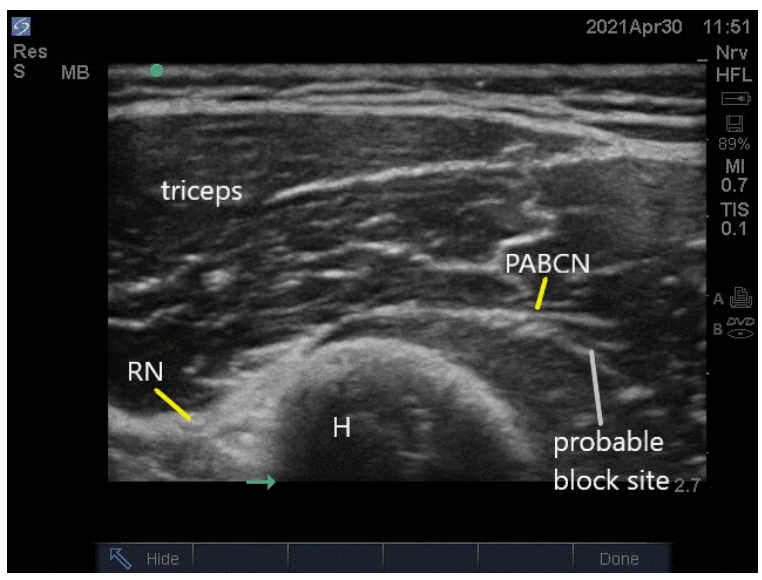

76 | International Journal of Regional Anaesthesia | Volume 2 | Issue 1 | January-June 2021 | Page $72-77$ 


\section{Conclusion}

The US-FANB's prove its worth in two clinical scenarios, (1) in the emergency department for early debridement of compound injuries to the hand and fingers [13] and (2) as a supplementation after an inadequate brachial plexus block [14] Apart from these continuous catheters can be inserted to treat chronic pain [15]. USFANB's should be considered as an important skill in the hands of regional anaesthesiologist.

\section{References}

1) Sayeh Hazmenzedeh, David Bravos. Peripheral Nerve Blocks of the Distal Upper Extremity.ASRA 2019.

2) Liebmann O, Price D, Mills C, Gardner R, Wang R, Wilson S, et al. Feasibility of forearm ultrasonography guided nerve blocks of the radial, ulnar, and median nerves for hand procedures in the emergency department. Ann Emerg Med 2006,48:558-62.

3) Jose Soberon, Neil Bhatt, Bobby Nossamon, Scott Duncan et al. Distal peripheral nerve blockade for patients undergoing hand surgery: a pilot study. Hand (New York)2015 Jun; 10(2): 197-204.

4) Herman Sehmbi, Caveh Madjdpour, Ushma Jitendra Shah, Ki Jinn Shin.Ultrasound guided distal peripheral nerve block of the upper limb: A technical review.JOACP 2015.Volume 31,issue3,296-307.

5) Jordan M.Brown, Corrie M. Yablon, Yoav Morag, Catherine J. Brandon, Jon A. Jacobson. Ultrasound of the Peripheral Nerves of the Upper Extremity: A Landmark Approach. RadioGraphics 2016; 36:452-463.

6) Chad Robertson, John Saratsiotis. A Review of Compressive Ulnar Neuropathy at the Elbow. Journal of manipulative and physiological therapeutics, June 2005;28(5):345.

7) Henrich Kele. Ultrasonography of the peripheral nervous system.

Perspectives in Medicine 2012.Volume 1, Issues 1-12, September 2012, Pages 417-421.

8) Admir Hadzic. Ultrasound-Guided Forearm Blocks.

Hadzic's Nerve Blocks and Anatomy for Ultrasound Guided Regional Anesthesia, 2e.
9) Wachara Wongkerdsook, Sithiporn Agthong, Chavarin Amarase, Pattarapol Yotnuengnit, Thanasil Huanmanop, Vilai Chentanez. Anatomy of the lateral antebrachial cutaneous nerve in relation to the lateral epicondyle and cephalic vein. Clinical Anat.Jan 2011;24(1):56-61.

10) Thomas K, Sajjad H, Bordoni B. Anatomy, Shoulder and Upper Limb, Medial Brachial Cutaneous Nerve. Treasure Island: Feb 14,2021.

11) Starr, Dennis Lee, Peter Stern. Anatomy of the Posterior Antebrachial Cutaneous Nerve, Revisited. Scientific Article Volume 45, Issue4, April 2020.

12) M Lurf et al. Ultrasound guided ulnar nerve catheter placement in the forearm for postoperative pain relief and physiotherapy.

Acta Anaesthesiology Scandinavia. Feb 2009;53(2):261-3.

13) Sanjeev Bhoi, Amit Chandra, Sagar Galwankar.

Ultrasound-guided nerve blocks in the emergency department

Journal of emergencies trauma and shock. Jan-Mar 2010.3(1):82-88.

14) RAMcCahon, NM Bedforth.Peripheral nerve blocks at the elbow

and wrist. Critical Care \& Pain journal, Volume 7, Issue 2, April 2007, Pages $42-44$.

15) Brian M Ilfeld. Continuous. Peripheral Nerve Blocks: An Update of the Published Evidence and Comparison With Novel, Alternative Analgesic Modalities. Anesth Analg, 2017Jan;124(1):308-335.

\section{Conflict of Interest: Nil \\ Source of Support: None}

77 | International Journal of Regional Anaesthesia | Volume 2 | Issue 1 | January-June 2021 | Page $72-77$ 